\title{
ZnS/Silica Nanocable Field Effect Transistors as Biological and Chemical Nanosensors
}

\author{
Jr H. He, ${ }^{*,+,,, \dagger}$ Yi Y. Zhang, ${ }^{\S}$ Jin Liu, ${ }^{\dagger}$ Daniel Moore, ${ }^{\dagger}$ Gang Bao, ${ }^{*, \S}$ and Zhong L. Wang, ${ }^{*, \dagger}$ \\ Graduate Institute of Electro-Optical Engineering and Department of Electrical Engineering, National Taiwan \\ University, Taipei, 106 Taiwan, School of Biomedical Engineering, Georgia Institute of Technology and Emory \\ University, Atlanta, Georgia 30332-0245, and School of Materials Science and Engineering, Georgia Institute \\ of Technology, Atlanta, Georgia 30332-0245
}

Received: June 19, 2007; In Final Form: July 13, 2007

\begin{abstract}
Compound semiconductor/isolator ( $\mathrm{ZnS} /$ silica) core/shell nanocables have been used to fabricate single nanowire-based field effect transistors. Using the surface-adsorbed charged molecules as the gate, the nanocablebased devices show potential for label-free, real-time, and sensitive detection of biological species. After chemical modification, amine- and oxide-functionalized nanocables exhibit linear $\mathrm{pH}$-dependent conductance, which could be elucidated in terms of the changes of surface charge during protonation and deprotonation. Selective biological recognition of nanocable sensors has been demonstrated using biotinylation.
\end{abstract}

One-dimension (1D) heterostructures such as superlattice nanowires $(\mathrm{NWs})^{1}$ and core-shell coaxial nanocables ${ }^{2}$ have attracted considerable interest due to their novel applications and properties in the unique integration of semiconductor/ semiconductor, ${ }^{1,2}$ conductor/semiconductor, ${ }^{3}$ isolator/semiconductor, ${ }^{4}$ and isolator/conductor nanosystems. ${ }^{5}$ The goal of the synthesis of $1 \mathrm{D}$ heterostructures at the nanoscale is to make multicomponent nanosystems that exhibit the unique merits offered by nanotechnology. Novel transistors consisting of GaN/ AlN/AlGaN nanowire heterostructures, for example, have been demonstrated for electronic nanodevices with high electron mobility. ${ }^{2 \mathrm{e}}$ Noble-metal nanoparticles embedded in dielectric matrices have potential applications in ultrafast optical switching nanodevices due to the enhanced third-order nonlinear susceptibility. ${ }^{5 a}$

Sensing using a planar field effect transistor (FET) has been demonstrated for decades, but lower sensitivity has limited its applications. ${ }^{6}$ Compared to the surface region of a planar device, due to the ultrahigh surface-to-volume ratio, $\mathrm{NWs}^{7}$ and carbon nanotubes $(\mathrm{CNTs})^{8}$ are ideal choices for sensors due to the introduced depletion/accumulation of charges near the surface as a result of surface binding/adsorption of foreign molecules and species. The NWs that have been extensively developed for sensing are silicon because of the massive knowledge that has existed for the chemical modification of native $\mathrm{Si}$ oxide surfaces. $^{2 \mathrm{e}, 9,10}$ The FET of $\mathrm{ZnS} /$ silica core/shell nanostructures are attractive to biological and chemical sensors due to (i) their excellent and reproducible electronic characteristics of $\mathrm{ZnS}$, (ii) the self-assembled silica shell as a natural insulator on the surface of the $\mathrm{ZnS}$ NW that serves as the protection layer against

* To whom correspondence should be addressed. E-mail: zhong.wang@ mse.gatech.edu (Z.L.W.); gang.bao@bme.gatech.edu (G.B.); jhhe@ cc.ee.ntu.edu.tw (J.H.H.).

$\doteqdot$ National Taiwan University.

$\S$ Georgia Institute of Technology and Emory University.

Georgia Institute of Technology. oxidation, and (iii) the massive existing knowledge about the chemical modification of silica surfaces.

In the present study, $\mathrm{ZnS} / \mathrm{SiO}_{2}$ core/shell nanocables have been used to fabricate a FET nanodevice. We demonstrated the reproducible transport characteristics of the nanocable FETs, in which metal electrode and electrolyte solutions were used as a gate. The nanocable-based devices were used for label-free, real-time, and sensitive detection of biological species. Amineand hydroxyl-functionalized $\mathrm{ZnS} /$ silica nanocables exhibit linear $\mathrm{pH}$-dependent conductance, which could be elucidated in terms of the changes of surface charge during protonation and deprotonation. Finally, selective biological recognition has been demonstrated after biotinylation of the nanocable's surface since the silica shell makes the receptor linkage straightforward. Exploratory research has been presented on the capability of $\mathrm{ZnS} /$ silica nanocables for label-free, real-time, sensitive, and selective detection of chemical and biological species.

The nanocables were synthesized using vapor-liquid-solid growth. Details about the growth, growth mechanism, and the formation process have been reported elsewhere. ${ }^{11}$ After the growth process, the substrate-bound nanocables were mechanically scrapped and sonicated in ethanol and deposited on silicacoated copper grids for transmission electron microscopy (TEM) characterization. A low-magnification TEM image of assynthesized nanocables shows uniformity in diameter (Figure 1a). Figure $1 \mathrm{~b}$ is a high-magnification TEM image which indicates that the nanostructure is a core-shell nanostructure. The corresponding selected area electron diffraction (SAED) from the shell of the nanocables, as shown in Figure 1c, confirms that the shell is amorphous. SAED from the core, as shown in Figure 1d, confirms that the phase is wurtzite-structured $\mathrm{ZnS}$. In TEM images, the contrast in $\mathrm{ZnS}$ core is related to the formation of basal-plane stacking faults, but the main body of the structure is wurtzite. ${ }^{12}$ An energy dispersive spectrometer (EDS) attached to the TEM was utilized to characterize the distribution of the chemical composition of the nanocables. Figure 1e shows that the amorphous shell consists of Si and O. 

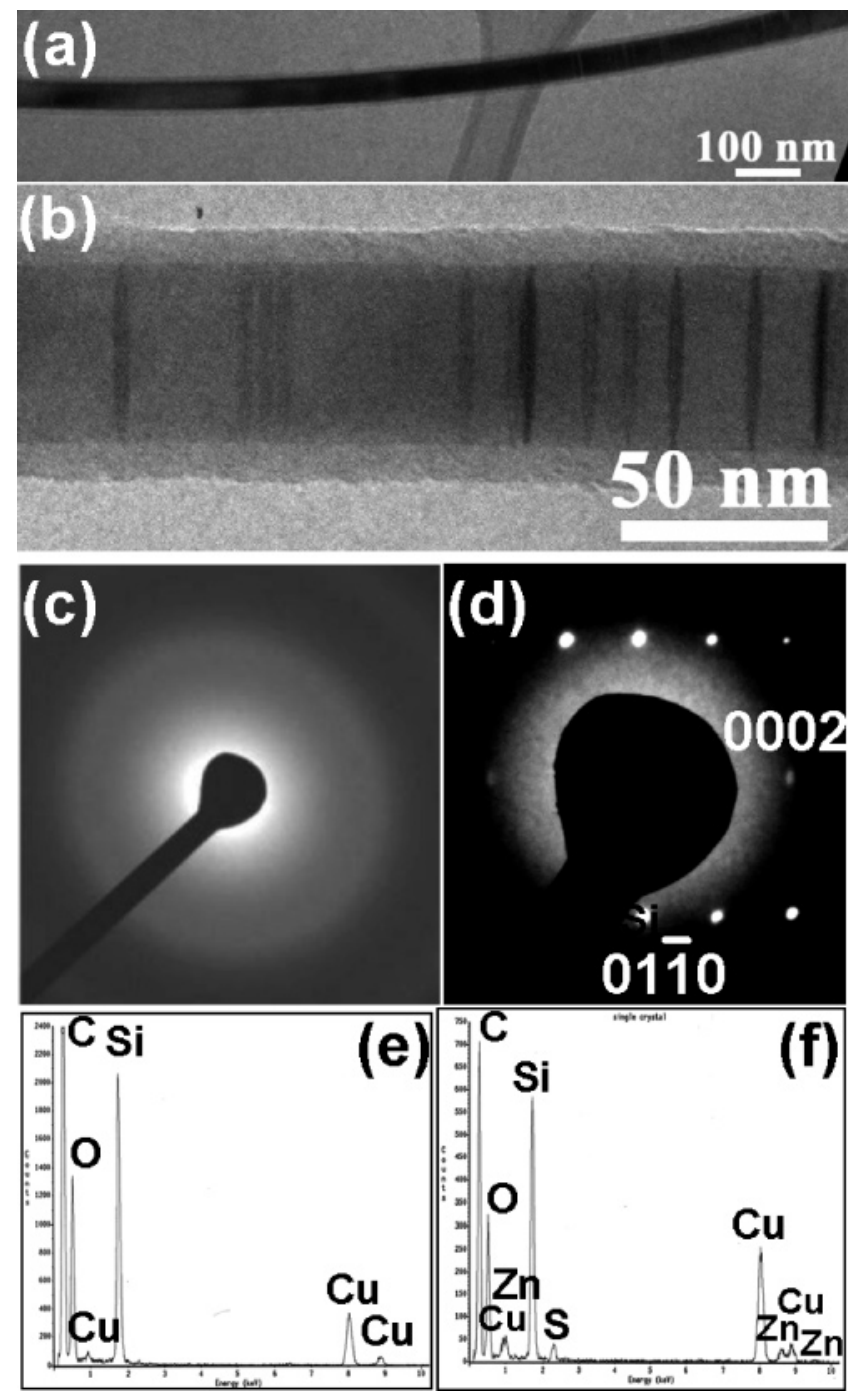

Figure 1. (a) Low-magnification and (b) high-magnification TEM image of a $\mathrm{ZnS} /$ silica nanocable. The corresponding SAED patterns from (c) the shell and (d) the core of the nanocables. The corresponding EDS analysis of (e) the shell and (f) the core of the nanocables.

Figure 1f shows that the core consists of $\mathrm{Zn}$ and $\mathrm{S}$. The higher signal of $\mathrm{Si}$ and $\mathrm{O}$ in Figure 1f might have resulted from the detection of a signal from silica-coated copper grids for TEM characterization. Therefore, the nanocable consists of a silica shell and a wurtzite-structured $\mathrm{ZnS}$ core.

For the fabrication of nanocable FETs, the electrode pattern was designed to have a few parallel electrodes separated by 5-20 $\mu \mathrm{m}$ using standard photolithography and a lift-off process. The synthesized $\mathrm{ZnS} /$ silica nanocables were transferred from the $\mathrm{Si}$ substrate to prepatterned $\mathrm{Au} / \mathrm{Ti}$ electrodes by touching the nanocable sample with the electrodes. A single nanocable device could be achieved easily using this method. To firmly contact the $\mathrm{Au} / \mathrm{Ti}$ metal electrodes and $\mathrm{ZnS}$ core of the nanocable, focused ion beam (FIB) microscopy was employed to cut the nanocable at the two ends so that the $\mathrm{ZnS}$ core was exposed, and then, a Pt mixture was deposited at the ends to make contacts between the $\mathrm{ZnS}$ core and Au/Ti electrodes. The oxygen plasma treatment was carried out to eliminate the surface contamination of the $\mathrm{ZnS} /$ silica nanocable device before biological and chemical sensing.

To assess the electrical characteristics of the $\mathrm{ZnS} / \mathrm{SiO}_{2}$ nanocables, FET devices were fabricated using standard procedures with back gate geometry. As shown in Figure 2a, the
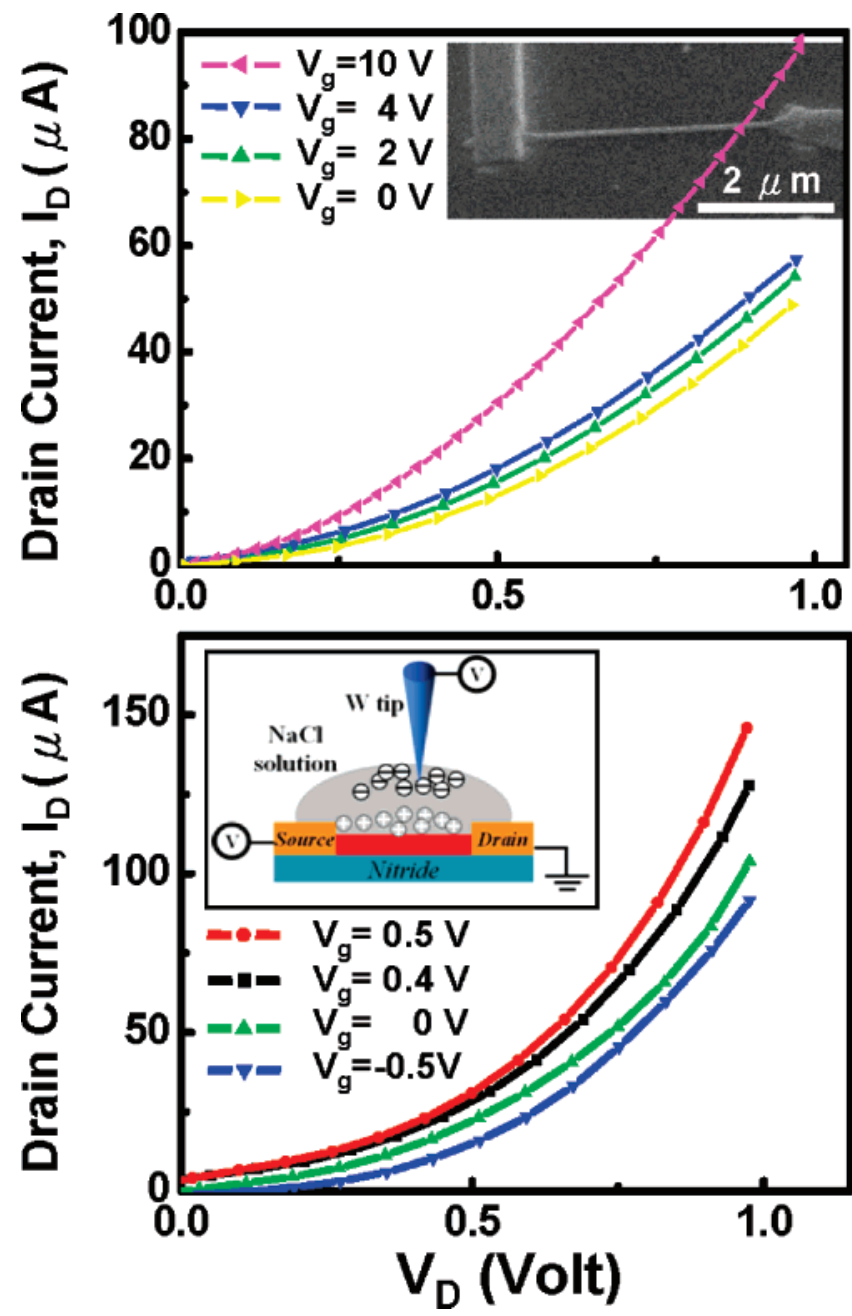

Figure 2. (a) $I_{\mathrm{D}}-V_{\mathrm{D}}$ measurements for a back-gate nanocable transistor. The inset is a typical SEM image of a nanocable transistor. (b) $I_{\mathrm{D}}-V_{\mathrm{D}}$ measurements for a nanocable-based transistor in a $3 \mu \mathrm{L}, 1 \times 10^{-3}$ $\mathrm{g} / \mathrm{L} \mathrm{NaCl}$ solution. The gate voltage is applied through a W electrode immersed in the solution, as shown in the inset.

drain current $\left(I_{\mathrm{D}}\right)$ versus drain voltage $\left(V_{\mathrm{D}}\right)$ characteristics of $\mathrm{ZnS} / \mathrm{SiO}_{2}$ nanocable-based transistors obtained as a function of different positive gate voltages $\left(V_{\mathrm{g}}\right)$ indicate that the pronounced gate effect is indicative of an n-type semiconductor. The inset in Figure $2 \mathrm{a}$ is a typical scanning electron microscopy (SEM) image of a nanocable FET. For electrical characterization of our devices in liquid, the chip was immersed into the electrolyte solution with an additional electrode, which allowed us to apply the gate voltage through the electrolyte solution. In the present study, we measured the current through FET using an electrolyte $\left(1 \times 10^{-3} \mathrm{M} \mathrm{NaCl}\right)$ as a gate, as schematically shown in the inset of Figure 2b. A micropipette was used to place a small electrolyte droplet $(3 \mu \mathrm{L})$ over the nanocable-based FETs. $V_{\mathrm{g}}$ applied to a $\mathrm{W}$ tip was used to establish the electrochemical potential in the electrolyte relative to the FET device. The $I_{\mathrm{D}}-$ $V_{\mathrm{D}}$ characteristics of the FET device, as shown in Figure 2b, were measured at different electrolyte-gated voltages. Sweeping $V_{\mathrm{g}}$ from negative to positive, resulting in the increase of $I_{\mathrm{D}}$, also shows the typical behavior for n-type FET devices. Evidently, a change in voltage of the liquid gate can produce a detectable change in drain current, leading to the potential of chemical and biological sensing in liquid.

Biological Sensing. Sensing in solution was carried out by monitoring electrical conductance through the $\mathrm{ZnS} /$ silica nanocable device during protein additions in phosphate buffer (PB) 


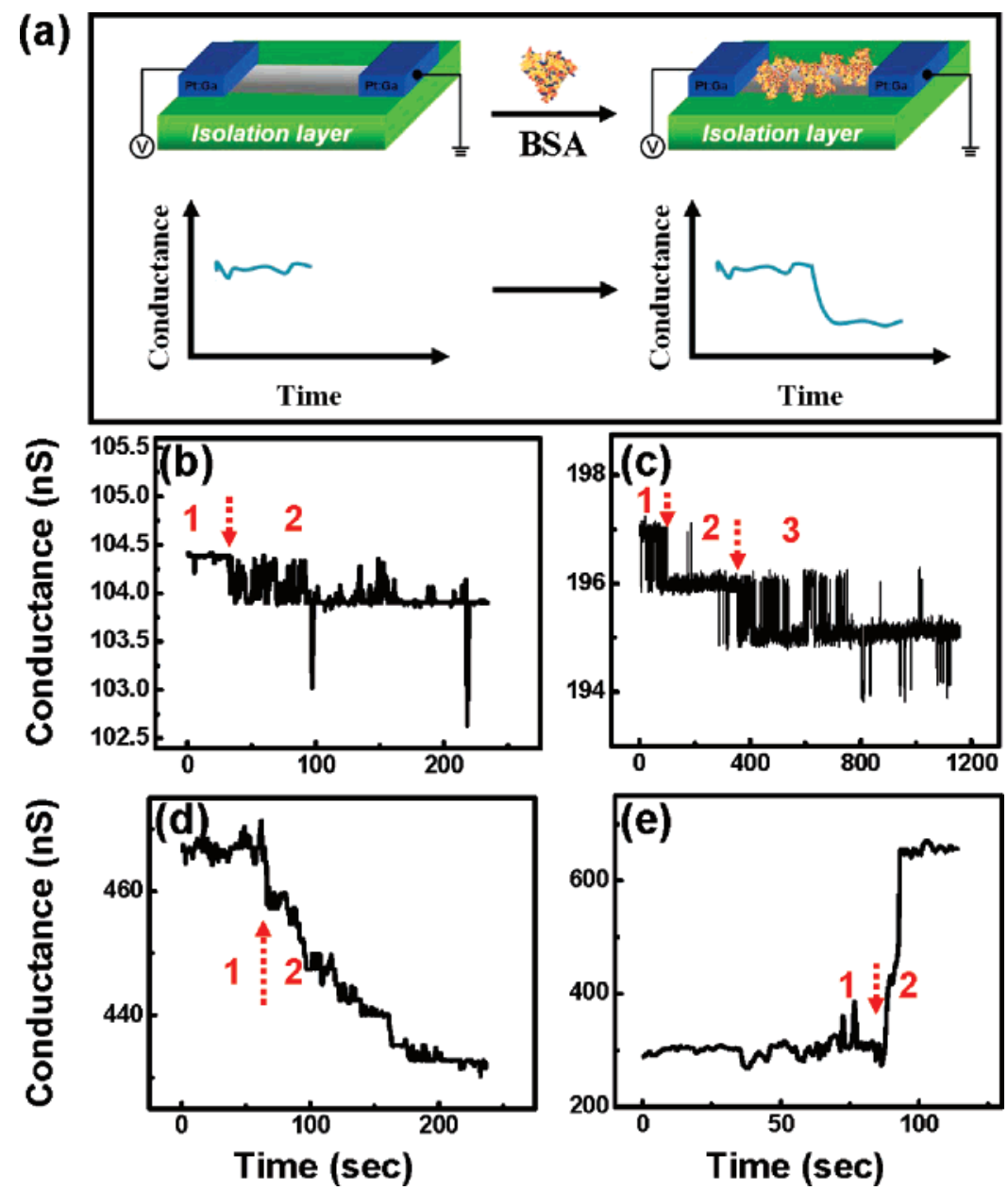

Figure 3. Real-time detection of nonspecific binding of protein. (a) Schematic diagram of a nanocable FET configured as a sensor; the binding of BSA with a net negative charge yields a decrease in the conductance. (b) Plot of conductance versus time of a nanocable device, where region 1 corresponds to the addition of $3 \mu \mathrm{L}$ of PB solution, and region 2 corresponds to the addition of $3 \mu \mathrm{L}$ of PB solution with $5 \times 10^{-4} \mathrm{~g} / \mathrm{L}$ BSA. (c) Plot of conductance versus time of a nanocable device during exposure to various concentrations of BSA solution, where region 1 corresponds to the addition of $3 \mu \mathrm{L}$ of PB solution with $5 \times 10^{-4} \mathrm{~g} / \mathrm{L} \mathrm{BSA}$, region 2 corresponds to the addition of $3 \mu \mathrm{L}$ of PB solution with $1 \times 10^{-3} \mathrm{~g} / \mathrm{L} \mathrm{BSA}$, and region 3 corresponds to the addition of $3 \mu \mathrm{L}$ of PB solution with $5 \times 10^{-3} \mathrm{~g} / \mathrm{L}$ BSA. (d) Plot of conductance versus time of a nanocable device, where region 1 corresponds to the addition of $3 \mu \mathrm{L}$ of PB solution, and region 2 corresponds to the addition of $3 \mu \mathrm{L}$ of $\mathrm{PB}$ solution with $1 \times 10^{-2}$ $\mathrm{g} / \mathrm{L}$ SA. (e) Plot of conductance versus time of a nanocable device, where region 1 corresponds to the addition of $3 \mu \mathrm{L}$ of $\mathrm{PB}$ solution, and region 2 corresponds to the addition of $3 \mu \mathrm{L}$ of $\mathrm{PB}$ solution with $1 \times 10^{-2} \mathrm{~g} / \mathrm{L}$ avidin.

solution. For example, bovine serum albumin (BSA) has a strong affinity for a variety of molecules binding to different sites, which makes possible the utilization of charged BSA attached to the surface of silica as a gate. The availability of the BSA on the surface of $\mathrm{ZnS} /$ silica nanocables was also measured by fluorescence microscopy. After cleaning in oxygen plasma to remove contaminants, $\mathrm{ZnS} /$ silica FETs were used for biological sensing.

The concept of our experiments is illustrated in Figure 3a. When nonspecific binding (NSB) of BSA to the surface of the nanocable device occurred, the conductance of that device changed from the baseline value. For an n-type nanocable, the conductance should decrease or increase when the net charge of the protein is correspondingly negative or positive. The negative charge on BSA with an isoelectric point (pI) of 4.8 is sufficient for the PB solution $(\mathrm{pH}=7.2)$. Moreover, NSB on the $\mathrm{ZnS} /$ silica nanocable is found to be a general phenomenon with all proteins studied in this work, including BSA, streptavitin (SA), and avidin. First, the nanocable-based transistor was immersed in a PB solution with BSA $\left(5 \times 10^{-4} \mathrm{~g} / \mathrm{L}\right)$, and its electrical conductance was decreased while proteins were added (Figure $3 b$ ). The conductance was decreased upon stepwise exposure to BSA $\left(5 \times 10^{-4}-5 \times 10^{-3} \mathrm{~g} / \mathrm{L}\right)$, strongly suggesting that adsorption of BSA on the surface of the nanocable is responsible for the observed conductance change, as shown in Figure 3c. It has been demonstrated that the detection lower limit for BSA is $5 \times 10^{-4} \mathrm{~g} / \mathrm{L}$. For further confirmation, the FET was immersed in a PB solution with SA $\left(1 \times 10^{-2} \mathrm{~g} / \mathrm{L}\right)$, leading to the decrease of conductance since negatively charged $\mathrm{SA}(\mathrm{pI}=5.5)$ could also act as a negative gate when the $\mathrm{pH}$ of the PB solution was 7.2, as shown in Figure 3d. In contrast, avidin $(\mathrm{pI}=10)$ could act as a positive gate when the $\mathrm{pH}$ of the PB solution was 7.2, resulting in the increase of conductance when the transistor was exposed to a PB solution with avidin $\left(1 \times 10^{-2} \mathrm{~g} / \mathrm{L}\right)$, as shown in Figure $3 \mathrm{e}$. It could be concluded that the binding of a positively charged protein on an n-type nanocable would induce an increase of conductance opposite to the conductance decrease of a negatively charged one, which means that the protein can provide an electrostatic gating effect. In addition, the measurement on reproducibility has been shown in Supporting Information. The real-time detection of BSA shows that this NSB is reversible, as the conductance exhibits recovery upon buffer rinsing.

Chemical Sensing. Chemical sensing in solution was carried out by monitoring electrical conductance through the surfacemodified $\mathrm{ZnS} /$ silica nanocable device during buffer solution 

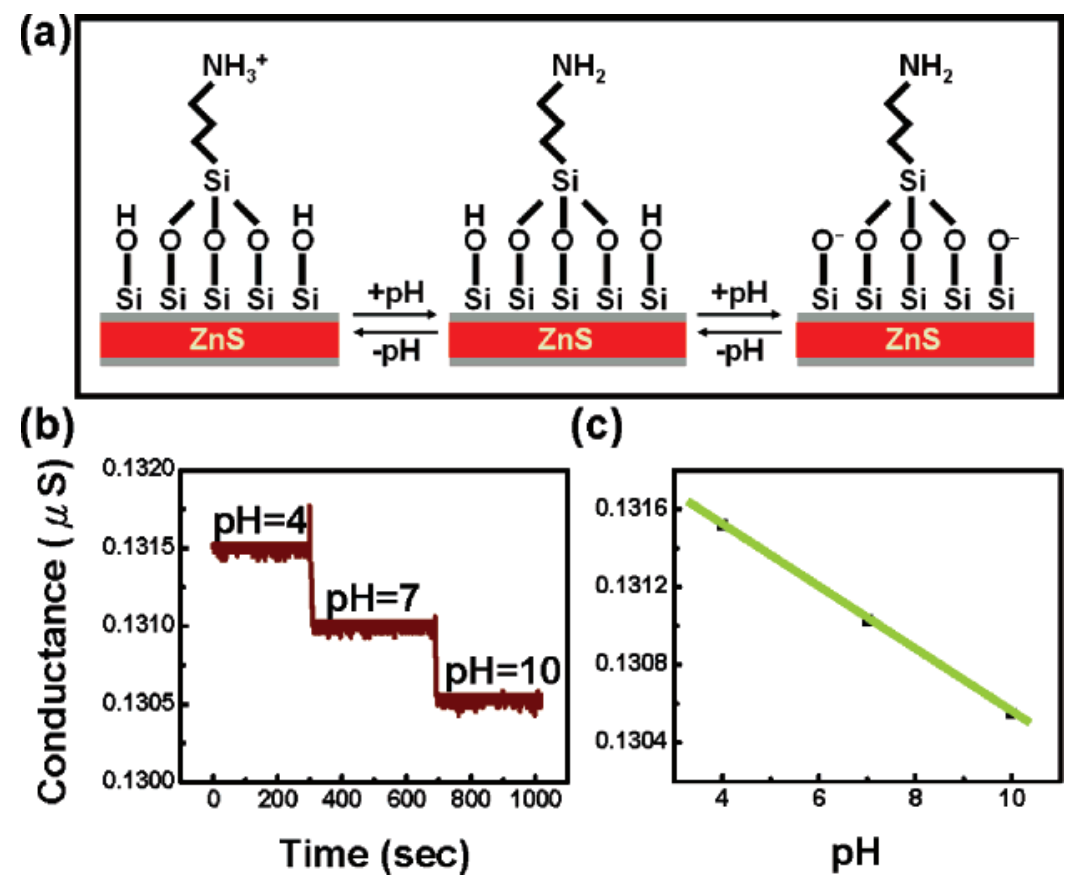

Figure 4. (a) Schematic illustrating a nanocable-based sensor for $\mathrm{pH}$ detection. The APTES-modified ZnS/silica nanocable changes in the surface charge state with $\mathrm{pH}$ due to $-\mathrm{NH}_{2}$ and $-\mathrm{SiOH}$ groups. (b) Real-time detection of the conductance for an APTE-modified nanocable; the $\mathrm{pH}$ values are indicated on the conductance plot. (c) Plot of the conductance versus $\mathrm{pH}$; the points are experimental data, and the solid line is a linear fit through the experimental data.
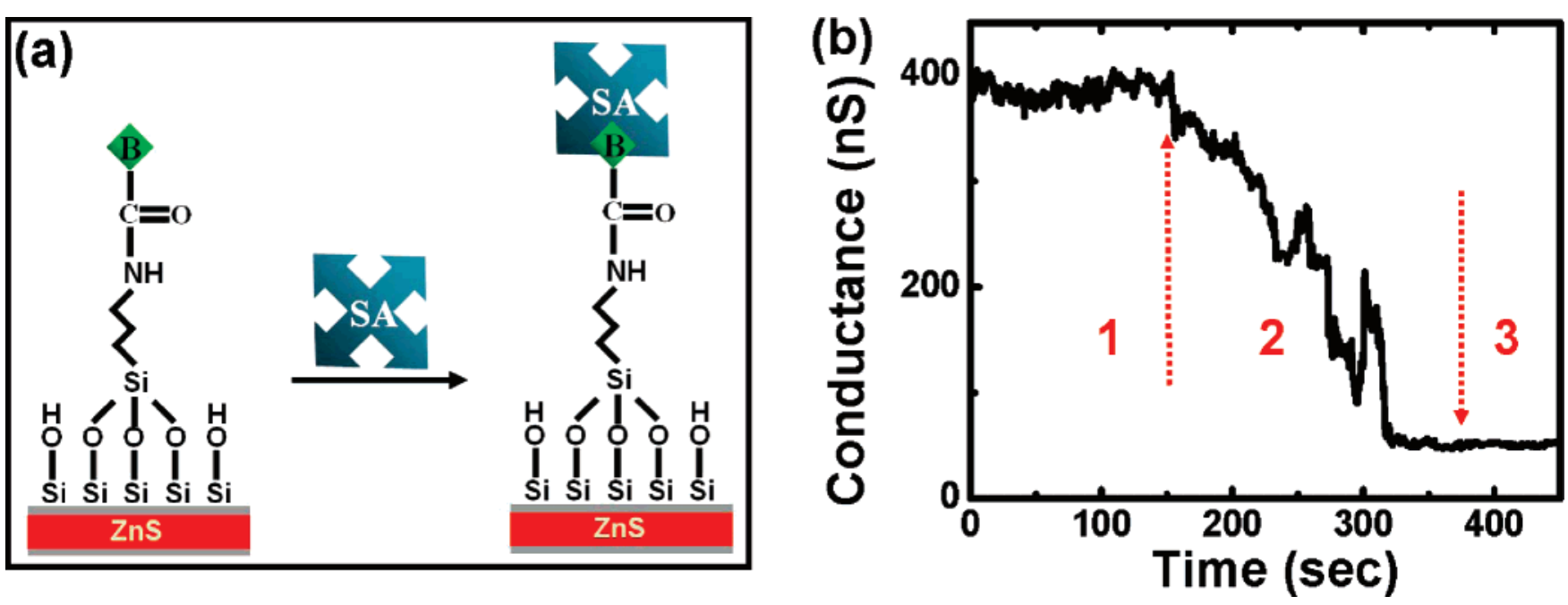

Figure 5. Real-time electrical detection of specific biological recognition. (a) Schematic diagram of a biotin-modified ZnS/silica nanocable (left) and subsequent binding of SA to the surface of the nanocable (right). (b) Conductance-time measurement for a biotin-modified nanocable, where region 1 corresponds to $3 \mu \mathrm{L}$ of $\mathrm{PB}$ solution, region 2 corresponds to the addition of $3 \mu \mathrm{L}$ of PB solution with $1 \times 10^{-2} \mathrm{~g} / \mathrm{L}$ SA, and region 3 corresponds to the addition of $3 \mu \mathrm{L}$ of PB solution with $1 \times 10^{-2} \mathrm{~g} / \mathrm{L}$ BSA.

additions with different $\mathrm{pH}$ values. Here, the $\mathrm{ZnS} /$ silica nanocable-based FET, whose conductance is modulated by an applied gate, is transformed into a chemical sensor by modifying the surface of the silica shell with 3-aminopropyltriethoxysilane (APTES) to provide a surface that can undergo protonation and deprotonation, where changes in the surface charge can chemically gate the nanocable-based transistors. After cleaning in oxygen plasma to remove contaminants, ZnS/silica FETs were placed in the chamber under the saturation vapor pressure of a $1 \%$ ethanol solution of APTES overnight and then rinsed with ethanol three times to achieve surface functionalization of nanocable FETs. The concept of a $\mathrm{pH}$ nanosensor is illustrated in Figure 4a. Measurements of conductance as a function of time and $\mathrm{pH}$ demonstrate that the NW conductance increases in steps corresponding to the $\mathrm{pH}$ values, as shown in Figure 4b. A representative curve of the conductance as a function of $\mathrm{pH}$ suggests that a modified $\mathrm{ZnS} /$ silica nanocable could be functionalized as a nanoscale $\mathrm{pH}$ sensor since its $\mathrm{pH}$ dependence is linear (Figure 4c). These results could be understood by considering the mixed surface functionality of the modified silica. The proposed mechanism for $\mathrm{pH}$ sensing is described as follows. Covalently linking APTES to the oxide surface of a nanocable results in a surface terminating in both $-\mathrm{NH}_{2}$ and $-\mathrm{SiOH}$ groups (Figure $4 \mathrm{a}$ ), which have different dissociation constants, $\mathrm{p} K_{\mathrm{a}}{ }^{7 \mathrm{a}, 13}$ At a high $\mathrm{pH}$ value, $-\mathrm{SiOH}$ is deprotonated to $-\mathrm{SiO}^{-}$on the surface of the silica shell and acts as a negative gate, which depletes electron carriers in the n-type $\mathrm{ZnS}$ core and decreases the conductance. At low $\mathrm{pH}$, the $-\mathrm{NH}_{2}$ group is protonated to $-\mathrm{NH}_{3}{ }^{+}$and acts as a positive gate, which correspondingly causes an increase in conductance. The ob- 
served linear response can be attributed to an approximately linear change in the total surface charge density due to the combined acid and base behavior of both surface groups. It is worth noting that the previous studies show that the conductance measurements exhibit a nonlinear $\mathrm{pH}$ dependence on the unmodified (only the $-\mathrm{SiOH}$ functionality) surface of silica. ${ }^{7 \mathrm{a}, 13 \mathrm{~b}}$

Specific Biological Recognition. Biological specificity, combined with the semiconducting properties of $\mathrm{ZnS}$, enables nanocable-based biosensors to selectively detect proteins in solution by using direct electronic readout without the need for labeling. To explore NW-based sensors for selective biological recognition, the silica shell of a nanocable was functionalized with biotin. The well-characterized ligand-receptor binding of SA-biotin (dissociation constant on the order of $10^{-15} \mathrm{M}$ ) would be utilized for specific recognition (Figure 5a). ${ }^{7 a, 14}$ The biontinylation reagent used here is amine-reactive in order to react with the APTES-modified $\mathrm{ZnS} /$ silica nanocable. The conductance-time measurement shows that the conductance of the biotin-modified nanocable decreases rapidly and considerably to a constant value upon addition of the SA solution, and this conductance value remains even after the addition of BSA solution (Figure 5b). Due to the small dissociation constant and small dissociation rate for biotin-SA, ${ }^{14}$ the absence of a conductance change with the addition of BSA was obtained. It demonstrates that nanocable-based biosensors are capable of specifically detecting proteins in real time, although the essentially irreversible biotin-SA binding interaction excludes real-time monitoring of varying protein concentrations.

In summary, using the semiconductor/isolator ( $\mathrm{ZnS} /$ silica) core/shell nanocable FETs and surface functionalization, we have shown that the FETs can be used for sensitive, label-free, real-time, selective, and electrical detection of chemical and biological species. This work presents the potential of coreshell-based nanowires for chemical and biochemical sensing.

Acknowledgment. Research was supported by EmoryGeorgia Tech CCNE (NIH), DARPA, AFOSR, AFRL, and NSC.

Supporting Information Available: Reproducibility results. This material is available free of charge via the Internet at http:// pubs.acs.org.

\section{References and Notes}

(1) (a) Wu, Y.; Fan, R.; Yang, P. D. Nano Lett. 2002, 2, 83-86. (b) Bjork, M. T.; Ohlsson, B. J.; Sass, T.; Persson, A. I.; Thelander, C.;
Magnusson, K.; Deppert, M. H.; Wallenberg, L.; Samuelson, L. Nano Lett. 2002, 2, 87-89. (c) Gudiksen, M. S.; Lauhon, L. J.; Wang, J.; Smith, D. C.; Lieber, C. M. Nature 2002, 415, 617-620.

(2) (a) Lauhon, L. J.; Gudiksen, M. S.; Wang, D. L.; Lieber, C. M. Nature 2002, 420, 57-61. (b) Chueh, Y. L.; Hsieh, C. H.; Chang, M. T. Chou, L. J.; Lao, C. S.; Song, J. H.; Gan, J. Y.; Wang, Z. L. Adv. Mater. 2007, 19, 143-149. (c) Hsu, Y. J.; Lu, S. Y.; Lin, Y. F. Adv. Funct. Mater 2005, 15, 1350-1357. (d) Manna, L.; Scher, E. C.; Li, L. S.; Alivisatos, A. P. J. Am. Chem. Soc 2002, 124, 7136-7145. (e) Li, Y.; Xiang, J.; Qian, F.; Gradecak, S.; Wu, Y.; Yan, H.; Blom, D. A.; Lieber, C. M. Nano Lett. 2006, 6, 1468-1473.

(3) Kong, X. Y.; Ding, Y.; Wang, Z. L. J. Phys. Chem. B 2004, 108 , $570-574$.

(4) (a) Chueh, Y. L.; Chou, L. J.; Wang, Z. L. Angew. Chem., Int. Ed. 2006, 45, 7773-7778. (b) Li, Y.; Ye, C. H.; Fang, X. S.; Yang, L.; Xiao, Y. H.; Zhang, L. D. Nanotechnology 2005, 16, 501-505. (c) Shen, G. Z; Bando, Y.; Tang, C. C.; Golberg, D. J. Phys. Chem. B 2006, 110, 71997202 .

(5) (a) Hu, M. S.; Chen, H. L.; Shen, C. H.; Hong, L. S.; Huang, B. R.; Chen, K. H.; Chen, L. C. Nature Mater. 2006, 5, 102-106. (b) He, J. H.; Wu, W. W.; Lee, S. W.; Chen, L. J.; Chueh, Y. L.; Chou, L. J. Appl. Phys. Lett. 2005, 86, 263109/1-263109/3.

(6) (a) Domansky, K.; Janata, J. Analyst 1993, 118, 335-340. (b) Janata, J. Analyst 1994, 119, 2275-2278.

(7) (a) Cui, Y.; Wei, Q.; Park, H.; Lieber, C. M. Science 2001, 293, 1289-1292. (b) Hahm, J.; Lieber, C. M. Nano Lett. 2004, 4, 51-54. (c) Patolsky, F.; Zheng, G. F.; Hayden, O.; Lakadamyali, M.; Zhuang, X. W.; Lieber, C. M. Proc. Natl. Acad. Sci. U.S.A. 2004, 101, 14017-14022. (d) Wang, W. U.; Chen, C.; Lin, K. H.; Fang, Y.; Lieber, C. M. Proc. Natl. Acad. Sci. U.S.A. 2005, 102, 3208-3212. (e) Zheng, G. F.; Patolsky, F.; Cui, Y.; Wang, W. U.; Lieber, C. M. Nat. Biotechnol. 2005, 23, 12941301. (f) Li, Z.; Chen, Y.; Li, X.; Kamins, T. I.; Nauka, K.; Williams, R. S. Nano Lett. 2004, 4, 245-247. (g) Bunimovich, Y. L.; Shin, Y. S.; Yeo, W. S.; Amori, M.; Kwong, G.; Heath, J. R. J. Am. Chem. Soc. 2006, 128, 16323-16331.

(8) (a) Zhou, C. W.; Kong, J.; Yenilmez, E.; Dai, H. J. Science 2000, 290, 1552-1555. (b) Chen, R. J.; Bangsaruntip, S.; Drouvalakis, K. A. Kam, N. W. S.; Shim, M.; Li, Y. M.; Kim, W.; Utz, P. J.; Dai, H. J. Proc. Natl. Acad. Sci. U.S.A. 2003, 100, 4984-4989. (c) Besteman, K.; Lee, J. O; Wiertz, F. G. M.; Heering, H. A.; Dekker, C. Nano Lett. 2003, 3, 727730. (d) Bradley, K.; Briman, M.; Star, A.; Gruner, G. Nano Lett. 2004, 4 253-256. (e) Li, C.; Curreli, M.; Lin, H.; Lei, B.; Ishikawa, F. N.; Datar, R.; Cote, R. J.; Thompson, M. E.; Zhou, C. W. J. Am. Chem. Soc. 2005 127, 12484-12485.

(9) Sze, S. M. Physics of Semiconductor Devices; John Wiley and Sons: New York, 1981.

(10) Liu, J.; Gao, P. X.; Mai, W. J.; Lao, C. S.; Wang, Z. L.; Tummala, R. Appl. Phys. Lett. 2006, 89, 063125/1-063125/3.

(11) Moore, S.; Morber, J. R.; Snyder, R. L.; Wang, Z. L. Small 2007, submitted.

(12) Ding, Y.; Wang, Z. L. J. Phys. Chem. B 2004, 108, 12280-12291.

(13) (a) Iler, R. K. The Chemistry of Silica; Wiley: New York, 1979 (b) Bolt, G. H. J. Phys. Chem. 1957, 61, 1166-1169. (c) Vezenov, D. V.; Noy, A.; Rozsnyai, L. F.; Lieber, C. M. J. Am. Chem. Soc. 1997, 119, 2006-2015.

(14) Wilchek, M.; Bayer, E. A. Methods Enzymol. 1990, 184, 49-51. 